\title{
Prosthetic heart valve assessment with multidetector-row CT: imaging characteristics of 91 valves in 83 patients
}

\author{
Jesse Habets • Petr Symersky • Lex A. van Herwerden • \\ Bas A. J. M. de Mol • Anje M. Spijkerboer • \\ Willem P. Th. M. Mali • Ricardo P. J. Budde
}

Received: 18 October 2010 /Revised: 26 November 2010 / Accepted: 12 December 2010 /Published online: 29 January 2011

(C) The Author(s) 2011. This article is published with open access at Springerlink.com

\begin{abstract}
Objectives Multidetector CT (MDCT) has shown potential for prosthetic heart valve (PHV) assessment. We assessed the image quality of different PHV types to determine which valves are suitable for MDCT evaluation.

Methods All ECG-gated CTs performed in our institutions since 2003 were reviewed for the presence of PHVs. After reconstruction in 3 specific PHV planes, image quality of the supravalvular, perivalvular, subvalvular and valvular regions was scored on a four-point scale $(1=$ non-diagnostic, $2=$ moderate, $3=$ good and $4=$ excellent) by two independent observers.

Results Eighty-four CT examinations (66 cardiac, 18 limiteddose aortic protocols) of 83 patients with a total of 91 PHVs in the aortic $(n=71)$, mitral $(n=17)$, pulmonary $(n=1)$ and
\end{abstract}

J. Habets $(\varangle) \cdot$ W. P. T. M. Mali $\cdot$ R. P. J. Budde

Department of Radiology, UMC Utrecht,

P.O. Box 85500, E01.132,

3508 GA Utrecht, The Netherlands

e-mail: J.Habets@umcutrecht.nl

L. A. van Herwerden

Department of Cardiothoracic Surgery,

University Medical Center Utrecht,

Utrecht, The Netherlands

P. Symersky

Department of Cardiothoracic Surgery, Onze Lieve Vrouwe Gasthuis, Amsterdam, The Netherlands

A. M. Spijkerboer

Department of Radiology, Academic Medical Center Amsterdam,

Amsterdam, The Netherlands

P. Symersky • B. A. J. M. de Mol

Department of Cardiothoracic Surgery,

Academic Medical Center Amsterdam,

Amsterdam, The Netherlands tricuspid $(n=2)$ position were included. CT was performed on a 16-slice $(n=4), 64$-slice $(n=28)$ or 256-slice $(n=52)$ MDCT system. Median image quality scores for the supra-, peri- and subvalvular regions and valvular detail were (3.5, $3.3,3.5$ and 3.5, respectively) for bileaflet PHV; (3.0, 3.0, 3.5 and 3.0, respectively) for Medtronic Hall PHV; (1.0, 1.0, 1.0 and 1.0, respectively) for Björk-Shiley and Sorin monoleaflet PHV and (3.5, 3.5, 4.0 and 2.0 respectively) for biological PHV.

Conclusion Currently implanted PHVs have good image quality on MDCT and are suitable for MDCT evaluation.

Keywords Prosthetic heart valve - Image quality $\cdot$ Heart valve $\cdot$ Cardiac imaging $\cdot$ Computed tomography

\section{Introduction}

Prosthetic heart valve (PHV) dysfunction is an uncommon complication after PHV implantation with potential lifethreatening consequences. In daily clinical practice, (suspected) PHV dysfunction is evaluated by the following non-invasive imaging techniques: transthoracic echocardiography (TTE), transesophageal echocardiography (TEE) and fluoroscopy [1]. These imaging techniques can detect PHV dysfunction accurately. However, echocardiography and fluoroscopy may fail to detect the anatomical substrate which causes PHV dysfunction [2]. Causes of PHV dysfunction include: pannus formation (subprosthetic tissue proliferation), thrombus formation, patient prosthesis mismatch (PPM, a too small valve for the patient's body size), pathologic (para)valvular leakage and endocarditis [1].

Recently, multidetector computed tomography (MDCT) has shown potential for non-invasive evaluation of PHV (dys)function. In small studies, MDCT demonstrated 
additional diagnostic value to echocardiography and fluoroscopy for the evaluation of PHV dysfunction, especially in detecting pannus tissue [3-6]. It is important to know which PHV types have a CT appearance that allows detection of PHV problems. However, little is currently known about the MDCT appearance of different valve types [3]. In our article titled "Multidetector-row computed tomography imaging characteristics of mechanical prosthetic valves" accepted for publication in Journal of Heart Valve Disease, we recently reported on imaging characteristics of five different PHV types in a controlled in-vitro model. The purpose of the current study was to assess the CT image quality of different PHV types in patients to determine which valves are suitable for $\mathrm{CT}$ evaluation.

\section{Materials and methods}

\section{CT selection}

We reviewed all ECG-gated $\mathrm{CT}(\mathrm{A}) \mathrm{s}$ performed in the University Medical Centre Utrecht (UMCU) and Academic Medical Centre Amsterdam (AMC) between 2003 and April 2010 for the presence of PHVs based on the presence of a PHV and/or steel wires through the sternum (indicating an previous median sternotomy) on the surview images. If one or both criteria were met the CT images were reviewed for the presence and type of PHV. Patients with only a mitral or tricuspid annuloplasty ring or a stented valvegraft as used in percutaneous valve replacement were excluded. All other PHV types were included. CT data, as well as the reason for requesting the CT examination, were retrieved from the PACS archive of the Radiology department. CT data was sent to a workstation for image analysis.

Heart rate during the examination was obtained from the CT data. Patient data on specific valve type and size were obtained from the patient medical files. The study was performed under a waiver from the local ethics committee.

Image analysis

Assessment of the CT examinations was performed on a dedicated workstation (Extended Brilliance Workstation, Philips Medical Systems, Philips, Best, the Netherlands). Three sets of images were reconstructed in three perpendicular imaging planes of each PHV: one set in plane with the valve, one set parallel and one set perpendicular to the valve leaflet(s). Reconstructions were made in both the diastolic and systolic phase of the cardiac cycle, if both phases were available.

For each PHV, image quality of the supravalvular, perivalvular, subvalvular and valvular region was scored on a fourpoint scale: $1=$ non-diagnostic, $2=$ moderate visualization,
$3=$ good visualization and $4=$ excellent visualization. The different regions are illustrated in Fig. 1.

The criteria for the different scores per region were formulated as follows: for the supra-, sub- and perivalvular region: 1: no discernible supra-, sub-, or perivalvular detail widely beyond prosthesis; 2 : no or limited details within $5 \mathrm{~mm}$ of prosthesis; 3: adequate details within $5 \mathrm{~mm}$ of prosthesis; 4 : perfect details. For valvular detail: 1 : no discernible leaflet; 2 : leaflet discernible but no angle measurements possible; 3 : leaflet angle measurement possible and 4: excellent leaflet detail. For the general score the image quality scores for all the regions were taken into account.

Scoring was performed by two observers (PS and JH) independent of each other. The observers had three and 1 year experience with cardiac $\mathrm{CT}$, respectively.

Data analysis

Data were analyzed using SPSS software (SPSS Statistics Version 15.0, SPSS Inc, Chicago, IL). The scores of the image quality scores per region of both observers was calculated and used for analysis. Data were presented as medians with minimum and maximum values because of a non-parametric data distribution. Image quality scores for different PHV types (bileaflet, tilting-disc and biological PHVs) and different manufacturers were compared with the Kruskal-Wallis test. If the Kruskal-Wallis test showed a significant result, the post-hoc Mann-Whitney $U$ test was performed with Bonferroni correction. Interobserver variability was analyzed by weighted kappa statistics based on Cohen. Correlations between image quality scores and heart rate, were analyzed using the Spearman correlation. The influence of the CT protocol (aortic CTA/dedicated cardiac CTA) or type of CT system (16/64/256 slice) on the image quality scores were analyzed using the Fisher's Exact Test. Statistical significance was defined as a p-value $<0.05$.

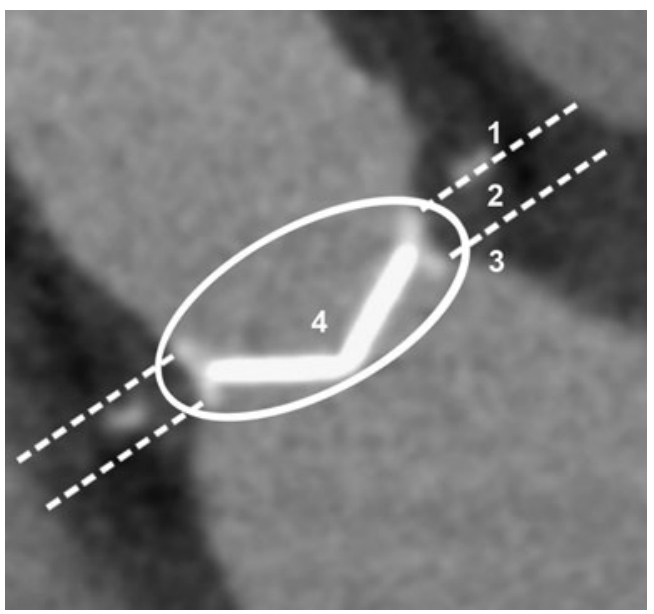

Fig. 1 Different valvular regions for MDCT assessment (1=supravalvular, $2=$ perivalvular, $3=$ subvalvular, $4=$ valvular detail) 
Table 1 Image quality (IQ) score specified per PHV manufacturer type

\begin{tabular}{|c|c|c|c|c|c|c|c|c|}
\hline \multirow[t]{2}{*}{ Type } & \multirow[t]{2}{*}{ Type } & \multirow[t]{2}{*}{ Manufacturer } & \multirow[t]{2}{*}{$\mathrm{N}=$} & \multicolumn{4}{|c|}{ Median IQ score (minimum-maximum) } & \multirow[t]{2}{*}{ General IQ score } \\
\hline & & & & Supravalvular & Perivalvular & Subvalvular & Valvular & \\
\hline \multirow{8}{*}{$\begin{array}{c}\text { Mechanical } \\
(n=70)\end{array}$} & \multirow{5}{*}{$\begin{array}{l}\text { Bileaflet } \\
\quad(n=50)\end{array}$} & Carbomedics & 20 & $3.8(2.5-4.0)$ & $3.5(2.0-4.0)$ & $3.5(2.5-4.0)$ & $3.5(2.0-4.0)$ & $3.5(1.5-4.0)$ \\
\hline & & St. Jude & 14 & $3.0(2.0-4.0)$ & $3.0(2.0-4.0)$ & $3.0(2.0-4.0)$ & $3.0(1.5-4.0)$ & $3.0(2.5-4.0)$ \\
\hline & & Sorin & 8 & $3.5(2.5-4.0)$ & $3.5(2.5-4.0)$ & $3.5(2.5-4.0)$ & $4.0(2.5-4.0)$ & $3.5(3.0-4.0)$ \\
\hline & & ON-X & 7 & $3.5(3.5-4.0)$ & $3.5(3.5-4.0)$ & $4.0(3.0-4.0)$ & $4.0(3.5-4.0)$ & $4.0(3.5-4.0)$ \\
\hline & & Duromedics & 1 & $3.0(3.0-3.0)$ & $2.0(2.0-2.0)$ & $2.5(2.5-2.5)$ & $3.0(3.0-3.0)$ & $2.5(2.5-2.5)$ \\
\hline & \multirow{9}{*}{$\begin{array}{l}\text { Tilting disc } \\
\quad(n=20)\end{array}$} & Medtronic Hall & 13 & $3.0(2.5-4.0)$ & $3.0(2.0-4.0)$ & $3.5(2.5-4.0)$ & $3.0(2.0-4.0)$ & $3.0(2.0-4.0)$ \\
\hline & & Björk-Shiley & 5 & $1.0(1.0-1.0)$ & $1.0(1.0-1.0)$ & $1.0(1.0-1.0)$ & $1.0(1.0-1.0)$ & $1.0(1.0-1.0)$ \\
\hline & & Sorin monoleaflet & 2 & $1.0(1.0-1.0)$ & $1.0(1.0-1.0)$ & $1.0(1.0-1.0)$ & $1.0(1.0-1.0)$ & $1.0(1.0-1.0)$ \\
\hline \multirow{6}{*}{$\begin{array}{l}\text { Biological } \\
\quad(n=21)\end{array}$} & & Perimount & 13 & $3.5(3.0-4.0)$ & $3.5(3.0-4.0)$ & $4.0(3.0-4.0)$ & $2.0(1.5-4.0)$ & $3.0(3.0-4.0)$ \\
\hline & & Medtronic intact & 2 & $3.5(3.0-4.0)$ & $3.3(3.0-3.5)$ & $3.3(2.5-4.0)$ & $1.5(1.0-2.0)$ & $3.0(2.5-3.5)$ \\
\hline & & Medtronic mosaic & 2 & $4.0(4.0-4.0)$ & $3.8(3.5-4.0)$ & $3.8(3.5-4.0)$ & $2.5(2.0-3.0)$ & $3.8(3.4-4.0)$ \\
\hline & & Mitroflow & 2 & $3.3(3.0-3.5)$ & $2.0(2.0-2.0)$ & $3.5(3.0-4.0)$ & $1.8(1.5-2.0)$ & $2.5(2.0-3.0)$ \\
\hline & & St. Jude Epic & 1 & $3.5(3.5-3.5)$ & $3.5(3.5-3.5)$ & $3.5(3.5-3.5)$ & $1.5(1.5-1.5)$ & $3.0(3.0-3.0)$ \\
\hline & & Freestyle & 1 & $4.0(4.0-4.0)$ & $4.0(4.0-4.0)$ & $4.0(4.0-4.0)$ & $2.0(2.0-2.0)$ & $4.0(4.0-4.0)$ \\
\hline
\end{tabular}

\section{Results}

Patient characteristics

A total of 84 ECG-gated examinations of 83 patients (age $56.3 \pm 14.8$ years $($ mean $\pm \mathrm{SD}$ ), 47 men) with 91 PHVs were available. Seventy mechanical PHVs and 21 biological PHVs were evaluated. One patient, who underwent two CTs, was included twice. This patient underwent a reoperation in which different PHVs were implanted between both CTs.

PHVs were positioned in the aortic $(n=71)$, mitral $(n=17)$, pulmonary $(n=1)$ and tricuspid position $(n=2)$. Seventy mechanical PHVs of 8 different manufacturers including 50 bileaflet PHVs (20 Carbomedics, 14 St Jude and 16 other PHVs) and 20 tilting disc valves (13 Medtronic Hall valves, 5 Björk-Shiley valves and 2 Sorin monoleaflet valves) were assessed. Twenty-one biological PHVs, 13 Perimount valves and eight other valves, were evaluated.

\section{CT parameters}

All patients underwent a retrospectively ECG-gated CT on a 16-slice $(n=4), 64$-slice $(n=28)$ or 256-slice $(n=52)$ MDCT system (Brilliance 16, Brilliance 64 and iCT, Philips Medical Systems, Cleveland, Ohio). The indications for the CT examinations were PHV or surrounding anatomy assessment $(n=42)$, evaluation of aortic aneurysms or dissection $(n=22)$ and other cardiac and pulmonary indications $(n=20)$. In $38 / 84(45 \%)$ of the patients the delay between surgery and CT could be inferred from the medical records. The median interval between surgery and the CT examination was 31 months (range: $0-175$ ). Mean heart rate during CT was $58 \pm 31 \mathrm{bpm}$ (range 48-139). In 13 of the 83 patients (16\%) the mean heart rate during CT was unknown. Sixty-six out of $84(79 \%)$ examinations were performed as a dedicated cardiac CT $(\geq 600 \mathrm{mAs})$ and $18 / 84$ (21\%) as an ECG-gated CT of the aorta (200-400 mAs). Fourty-two of the $66(64 \%)$ dedicated cardiac CT examinations were performed assessed for diagnostic PHV

Table 2 Image quality (IQ) score specified per PHV design type

\begin{tabular}{|c|c|c|c|c|c|c|}
\hline \multirow[t]{2}{*}{ Type } & \multirow[t]{2}{*}{$\mathrm{N}=$} & \multicolumn{4}{|c|}{ Median IQ score (minimum-maximum) } & \multirow[t]{2}{*}{ General IQ score } \\
\hline & & Supravalvular & Perivalvular & Subvalvular & Valvular & \\
\hline Bileaflet & 50 & $3.5(2.0-4.0)$ & $3.3(2.0-4.0)$ & $3.5(2.0-4.0)$ & $3.5(1.5-4.0)$ & $3.5(2.5-4.0)$ \\
\hline Tilting disc & 20 & $3.0(1.0-4.0)$ & $2.5(1.0-4.0)$ & $2.8(1.0-4.0)$ & $2.5(1.0-4.0)$ & $2.8(1.0-4.0)$ \\
\hline Biological & 21 & $3.5(3.0-4.0)$ & $3.5(2.0-4.0)$ & $4.0(2.5-4.0)$ & $2.0(1.0-4.0)$ & $3.0(2.0-4.0)$ \\
\hline
\end{tabular}


Fig. 2 St. Jude PHV in aortic position. The left image panel shows the excellent PHV visualization. The arrow head in the detailed right image panel indicates a PTFE pledget (component of the suture material) commonly used in PHV implantation
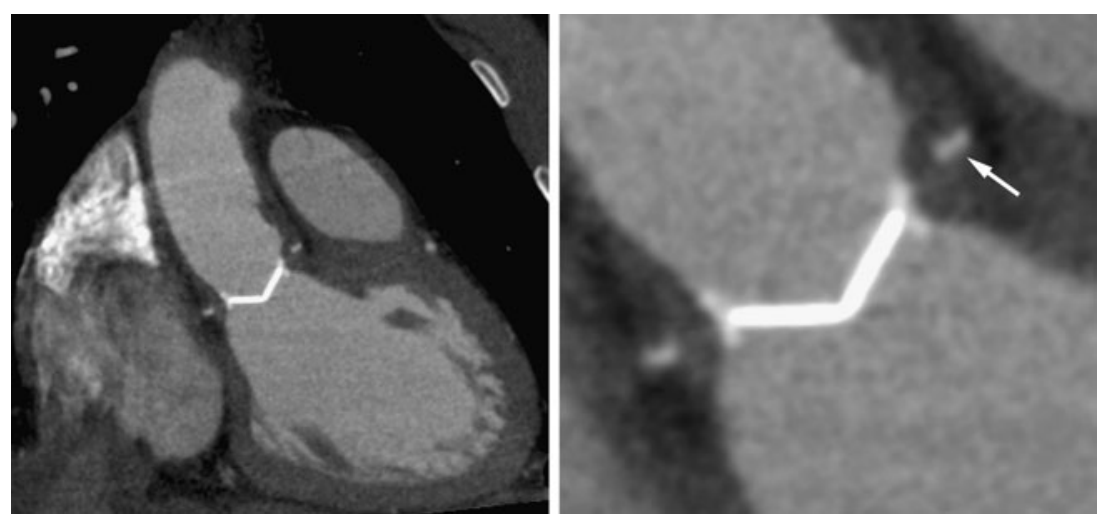

purposes. Contrast agents (Ultravist ${ }^{\circledR}-300 \mathrm{mg}$ jopromide/ $\mathrm{ml}$, Bayer Schering Pharma AG, Berlin, Germany or Imeron ${ }^{\circledR}-400 \mathrm{mg}$ iodine/ml, Bracco UK Limited, London, United Kingdom) were administered in all patients except in one patient with a Björk-Shiley PHV.

\section{Image quality scores}

The median image quality scores per PHV manufacturer type and PHV design type are shown in Tables 1 and 2. MDCT characteristics of different PHVs are illustrated in Figs. 2, 3, 4, 5 and 6.

Comparing bileaflet PHVs with tilting disc PHVs demonstrated significant higher image quality scores for the bileaflet valves for all regions $(p<0.001)$. Nevertheless, the Medtronic Hall tilting disc demonstrated no significant differences in image quality scores for all regions compared with the bileaflet PHVs. The comparison of bileaflet PHVs versus biological PHVs resulted in no significant differences in image quality per region, except for the valvular detail which was significantly higher for bileaflet valves $(p<0.001)$. The comparison of tilting disc PHVs versus biological PHVs resulted in significant $(p<0.001)$ higher image quality scores for the biological PHVs except for the valvular detail which did not differ significantly. In the tilting disc group, the image quality scores for all regions of the Medtronic Hall PHVs were significantly higher than for the Björk-Shiley PHVs and the Sorin monoleaflet PHVs $(p=0.001)$. In the biological and the bileaflet group, no significant differences in image quality scores were present for the different PHV manufacturers. No significant correlation between heart rate and the general image quality score was found $(p=0.635)$. Furthermore, the use of different $\mathrm{CT}$ systems showed no significant differences for general image quality scores ( $p=0.889,64$ vs. 256-slice, 16-slice CT systems excluded from calculation because only $4 \mathrm{CT}$ examinations available). Different CT protocols (aortic CTA vs dedicated cardiac CTA) demonstrated no significant difference in general image quality score $(p=0.629)$.

Interobserver variability

The weighted kappa value for the image quality scores of both observers was 0.79 with a standard error of 0.10 . This indicates a good interobserver agreement

\section{Discussion}

The principal results of this study are: (1) currently implanted PHVs and the periprosthetic region can be visualized with at least a good image quality by MDCT; (2) Björk-Shiley and Sorin monoleaflet tilting disc valves
Fig. 3 C-E Perimount PHV in aortic position. In the left image panel (long axis view), the artefacts caused by the metallic components of the struts are visualised. In particular in the supravalvular region. In the right image panel (the short axis view), the valve leaflets are well visualised
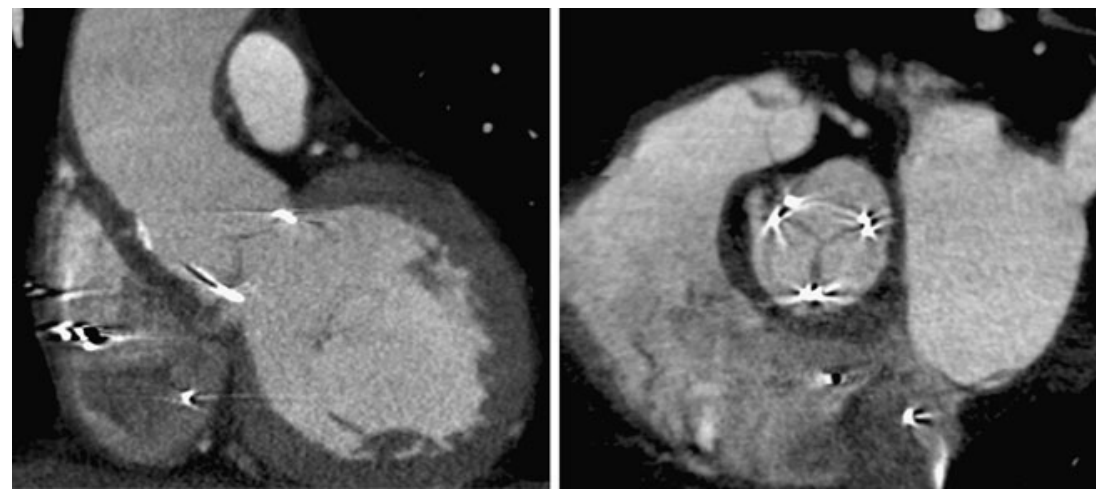


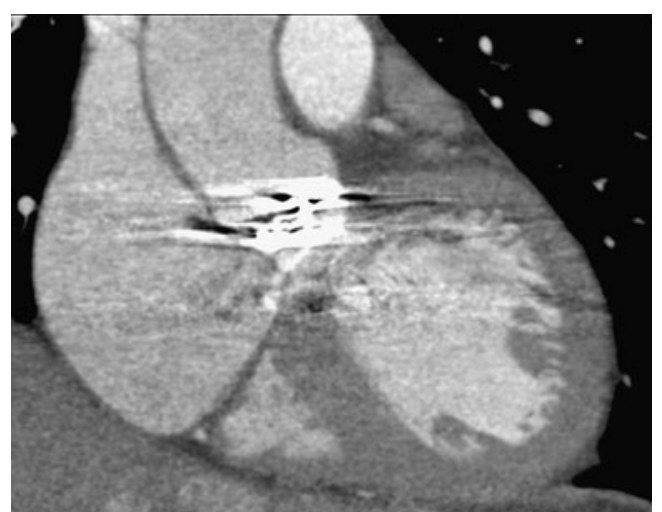

Fig. 4 Björk-Shiley tilting disc in aortic position. This PHV type and the periprosthetic anatomy are not suitable for MDCT diagnostic assessment due to severe artefacts

have significantly lower image quality than other PHVs and demonstrate severe artefacts which preclude diagnostic assessment.

TTE, TEE and fluoroscopy are the primary imaging modalities used for PHV assessment [1]. Echocardiography provides both anatomical and functional information. Fluoroscopy provides information on the leaflet motion in patients with a mechanical PHV only. These imaging techniques are suitable to detect PHV dysfunction, but may fail to detect the pathologic anatomical substrate, especially if this is pannus tissue [2]. Girard et al. [2] evaluated a total of 92 patients that underwent reoperation for prosthetic aortic valve obstruction: 49 mechanical and 43 biological PHVs. In the mechanical PHV group, the mechanism of obstruction (pannus, thrombosis and PPM) was correctly identified by TTE and TEE in 5/49 (10\%) and 17/35 (49\%) of cases, respectively. In the biological PHV group, the mechanism of aortic valve obstruction was correctly diagnosed in $27 / 4363 \%$ (TTE) and 21/26 81\% (TEE) of the cases, respectively. These findings illustrate that a conventional work-up of PHV dysfunction with echocardiography fails to detect the cause of dysfunction in upto $51 \%$ of mechanical PHVs.

MDCT is emerging as a new diagnostic modality to evaluate PHV dysfunction. Figure 7 illustrates the potential diagnostic potential of MDCT in visualizing PHV dysfunction.
We previously reported our findings in 13 patients with 15 mechanical heart valves. MDCT imaging provided additional diagnostic findings to echocardiography and fluoroscopy in 9 of 13 patients (69\%) [4]. MDCT is especially suitable to visualize pannus formation [5]. Our findings are supported by other groups. Tsai et al. [6] reported on 25 patients with 31 PHVs evaluated by TTE and MDCT. In 6 patients who underwent reoperation, MDCT findings were confirmed by intraoperative surgical findings. The image quality of PHVs was good, except for one Björk-Shiley tilting disc valve. In this prospective study diagnostic evaluation with TEE and fluoroscopy were lacking. Because some PHV types present with severe artefacts on $\mathrm{CT}$, it is important to determine which PHV types are suitable for CT assessment. Data concerning the CT compatibility of PHV are scarce. Konen et al. [3] assessed leaflet motion in 20 patients with 23 PHVs using a 40- or 64-MDCT. This study included 18 bileaflet and 5 tilting disc valves. The image quality of the bileaflet mechanical valves was good to excellent whereas the image quality of the tilting disc valves (Björk-Shiley and Sorin monoleaflet PHVs) was significantly lower. In only two out of the five tilting disc valves the opening and closing angles could be measured. Tsai et al.[6] evaluated two tilting disc valves (Medtronic Hall and Björk-Shiley tilting disc) and found severe artefacts in the Björk-Shiley valve precluding diagnostic assessment. In our article titled "Multidetector-row computed tomography imaging characteristics of mechanical prosthetic valves" accepted for publication in the Journal of Heart Valve Disease, we examined the CT imaging characteristics of 5 different PHV types in a strictly controlled in vitro test set-up with valve leaflet motion but no annular motion. In this study modern PHVs manufactured of carbon and titanium (St Jude, ON-X, Medtronic Hall, Carbomedics) showed a good image quality. The Björk-Shiley valve, made of a cobalt chrome alloy, exhibited severe artefacts. Within the carbon titanium group, the Carbomedics and $\mathrm{ON}-\mathrm{X}$ valve scored better than the St Jude and Medtronic Hall valves. These differences were attributed to valve design. Although this study demonstrated the suitability of CT for the assessment of PHV, the clinical application of CT could well
Fig. 5 Carbomedics mechanical $\mathrm{PHV}$ in aortic position
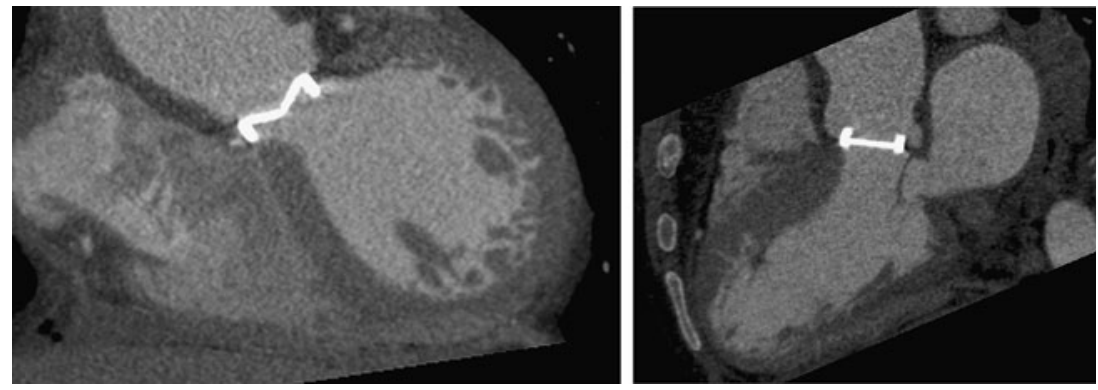
Fig. 6 Medtronic Hall mechanical PHV in mitral position
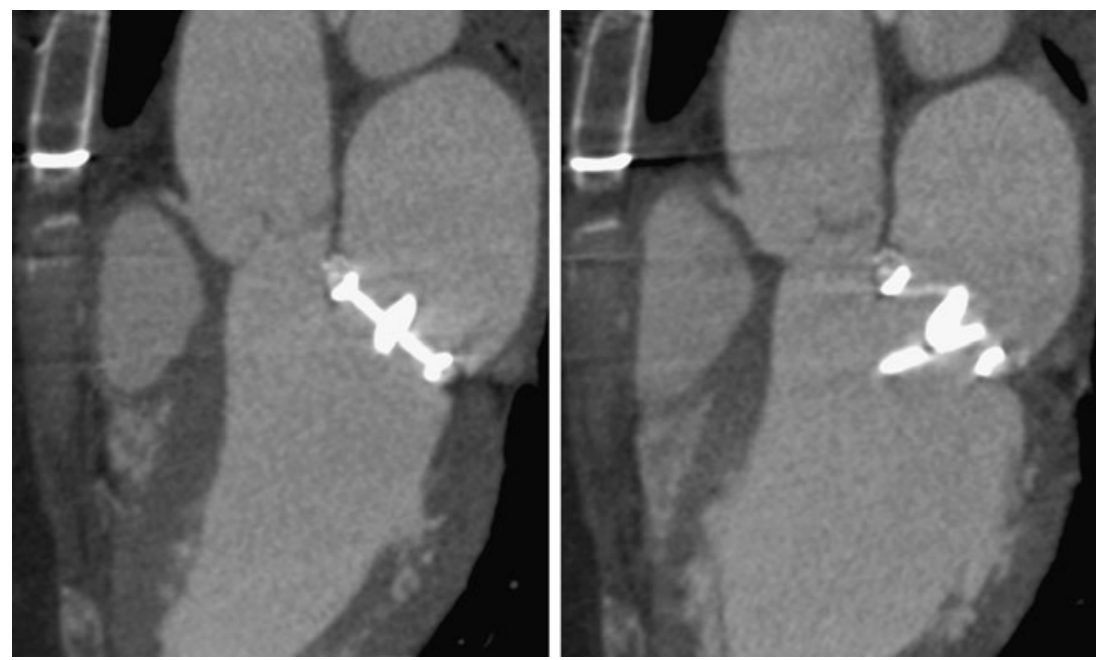

be compromised by motion artefacts and rhythm irregularities. The current study was undertaken to systematically examine the clinical performance of $\mathrm{CT}$, and, to our knowledge, is the largest series of PHVs assessed by CT described to date.

In addition to the 5 PHVs tested in vitro, we currently evaluated 9 additional PHV types covering a whole range of mechanical and biological PHV. For the mechanical PHV, our findings reflected the in vitro experience: we found that the Medtronic Hall valve had a significantly better image quality than Björk-Shiley and Sorin tilting disc valves. Furthermore, the Carbomedics and $\mathrm{ON}-\mathrm{X}$ valves had a better image quality than the St Jude and Medtronic Hall valves (Table 1). However, this finding was not significant. This discrepancy in image quality can be related to the differences in PHV design. The assessment of the

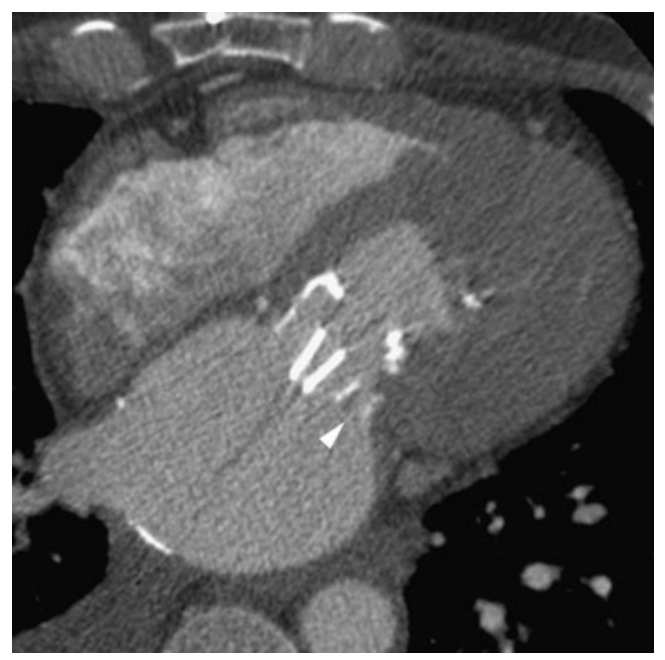

Fig. 7 PHV dysfunction on MDCT. A St Jude PHV in the mitral position with paravalvular leakage (arrowhead)
Björk-Shiley and the Sorin monoleaflet tilting disc valves resulted in non-diagnostic image quality of all scoring regions due to severe artefacts. These artefacts are the result of the presence of cobalt in the valve housing and strut mechanism. In clinical practice, these cobalt containing PHVs are not suitable for evaluation with $\operatorname{MDCT}[3,4,6]$. All currently implanted mechanical PHVs can be assessed by MDCT because they are all manufactured of titanium and carbon.

The biological PHVs show a good image quality except for valvular detail. We scored diastolic images and found that the leaflets of several bioprostheses could not be identified properly. We postulate that the limitations in spatial and temporal resolution of the CT may preclude good image quality in bioprostheses with thin leaflets (porcine aortic valve leaflets). Our results were less promising than a recent report from Chenot et al. [7] that demonstrated the feasibility of planimetric measurement of the orifice area of biological prostheses. The primary cause for this disparity may well be that our series was purely retrospective and that not all scans were specifically made for imaging of the bioprosthesis. Six of the 21 (29\%) biological PHVs were examined using a CT protocol for aortic imaging that uses a lower mAs setting which may have hampered the visualisation of the valve leaflets. The differences in contrast and imaging protocols may have resulted in a superior image quality in Chenot's series. Furthermore, Chenot et al. [7] identified several modes of biological PHV dysfunction such as leaflet thickening, calcification and possible thrombus. Leaflet thickening is likely to make the leaflets more easily discernable on CT. However, the retrospectively ECGgated scans that allow for dynamic assessment of PHV are associated with a relative high radiation dose. Lower dose PHV CT protocols are currently being explored [8].

Continued efforts for large scale prospective studies are required to further determine (1) normal MDCT characteristics of commonly implanted PHV types, (2) the exact 
additional diagnostic value of MDCT in evaluation of PHV dysfunction, (3) and the best imaging protocols for PHV with special attention to dose reduction.

\section{Limitations}

The study has a retrospective study design. In addition to dedicated cardiac CT, 18 ECG-gated aortic CT studies with a lower mAs setting were included. The lower mAs setting may have negatively influenced image quality. However, the aortic scan protocol was not associated with a significant lower image quality score. Some PHVs were present in small numbers. However, MDCT scans of the currently most commonly implanted PHVs (Carbomedics, St Jude, ON-X, Sorin and Perimount) were available for at least 7 valves each. Despite a mean heart rate of $58 \mathrm{bpm}$ during scanning there was a relatively wide range of heart rates (48-139). Although heart rates were not significantly correlated with a lesser CT image quality in our series, motion artefacts at higher heart rates may decrease image quality. In addition, aggressive heart rate lowering using beta blockers during scanning is contraindicated in some PHV patients as they may have poor left ventricular function and conduction abnormalities.

Although no correlation between image quality and heart rate, type of $\mathrm{CT}$ system and $\mathrm{CT}$ protocol were found, it should be noted that this retrospective study was not specifically designed for this.

\section{Conclusion}

MDCT is a promising imaging technique to evaluate PHV dysfunction. Currently implanted PHVs generate only limited artefacts and are suitable for evaluation with MDCT. CT assessment of Björk-Shiley and Sorin monoleaflet tilting disc PHVs is hampered by severe artefacts.

Acknowledgements The present study was supported by a grant of the Netherlands Heart Foundation [Grant number 2009B014]

Open Access This article is distributed under the terms of the Creative Commons Attribution Noncommercial License which permits any noncommercial use, distribution, and reproduction in any medium, provided the original author(s) and source are credited.

\section{References}

1. Zoghbi WA, Chambers JB, Dumesnil JG, Foster E, Gottdiener JS, Grayburn PA, Khandheria BK, Levine RA, Marx GR, Miller FA Jr, Nakatani S, Quinones MA, Rakowski H, Rodriguez LL, Swaminathan M, Waggoner AD, Weissman NJ, Zabalgoitia M (2009) Recommendations for evaluation of prosthetic valves with echocardiography and doppler ultrasound: a report From the American Society of Echocardiography's Guidelines and Standards Committee and the Task Force on Prosthetic Valves, developed in conjunction with the American College of Cardiology Cardiovascular Imaging Committee, Cardiac Imaging Committee of the American Heart Association, the European Association of Echocardiography, a registered branch of the European Society of Cardiology, the Japanese Society of Echocardiography and the Canadian Society of Echocardiography, endorsed by the American College of Cardiology Foundation, American Heart Association, European Association of Echocardiography, a registered branch of the European Society of Cardiology, the Japanese Society of Echocardiography, and Canadian Society of Echocardiography. J Am Soc Echocardiogr 22:975-1014

2. Girard SE, Miller FA Jr, Orszulak TA, Mullany CJ, Montgomery S, Edwards WD, Tazelaar HD, Malouf JF, Tajik AJ (2001) Reoperation for prosthetic aortic valve obstruction in the era of echocardiography: trends in diagnostic testing and comparison with surgical findings. J Am Coll Cardiol 37:579-584

3. Konen E, Goitein O, Feinberg MS, Eshet Y, Raanani E, Rimon U, Di-Segni E (2008) The role of ECG-gated MDCT in the evaluation of aortic and mitral mechanical valves: initial experience. Am J Roentgenol 191:26-31

4. Symersky P, Budde RPJ, de Mol BAJM, Prokop M (2009) Comparison of multidetector-row computed tomography to echocardiography and fluoroscopy for evaluation of patients with mechanical prosthetic valve obstruction. Am J Cardiol 104:1128-1134

5. Teshima H, Hayashida N, Fukunaga S, Tayama E, Kawara T, Aoyagi S, Uchida M (2004) Usefulness of a multidetector-row computed tomography scanner for detecting pannus formation. Ann Thorac Surg 77:523-526

6. Tsai IC, Lin YK, Chang Y, Fu YC, Wang CC, Hsieh SR, Wei HJ, Tsai HW, Jan SL, Wang KY, Chen MC, Chen CC (2009) Correctness of multi-detector-row computed tomography for diagnosing mechanical prosthetic heart valve disorders using operative findings as a gold standard. Eur Radiol 19:857-867

7. Chenot F, Montant P, Goffinet C, Pasquet A, Vancraeynest D, Coche E, Vanoverschelde JL, Gerber BL (2010) Evaluation of anatomic valve opening and leaflet morphology in aortic valve bioprosthesis by using multidetector CT: comparison with transthoracic echocardiography. Radiology 255:377-385

8. Symersky P, Budde RPJ, Prokop M, de Mol BAJM (2009) Abstract 541: prosthetic valve evaluation using prospective triggering with 256-detector row computed tomography reduces radiation dose. Circulation 120:S355 